\title{
ZERO LIE PRODUCT DETERMINED BANACH ALGEBRAS, II
}

\author{
J. ALAMINOS, M. BREŠAR, J. EXTREMERA, AND A.R. VILLENA
}

\begin{abstract}
A Banach algebra $A$ is said to be zero Lie product determined if every continuous bilinear functional $\varphi: A \times A \rightarrow \mathbb{C}$ satisfying $\varphi(a, b)=0$ whenever $a b=b a$ is of the form $\varphi(a, b)=\omega(a b-b a)$ for some $\omega \in A^{*}$. We prove that $A$ has this property provided that any of the following three conditions holds: (i) $A$ is a weakly amenable Banach algebra with property $\mathbb{B}$ and having a bounded approximate identity, (ii) every continuous cyclic Jordan derivation from $A$ into $A^{*}$ is an inner derivation, (iii) $A$ is the algebra of all $n \times n$ matrices, where $n \geq 2$, over a cyclically amenable Banach algebra with a bounded approximate identity.
\end{abstract}

\section{INTRODUCTION}

Let $A$ be a Banach algebra. For $a, b \in A$, we will write $[a, b]=a b-b a$ and $a \circ b=a b+b a$. Let $\varphi: A \times A \rightarrow \mathbb{C}$ be a continuous bilinear functional satisfying

$$
a, b \in A,[a, b]=0 \Longrightarrow \varphi(a, b)=0 .
$$

This is certainly fulfilled if $\varphi$ is of the form

$$
\varphi(a, b)=\omega([a, b]) \quad(a, b \in A)
$$

for some $\omega$ in $A^{*}$, the dual of $A$. We say that $A$ is a zero Lie product determined Banach algebra if, for every continuous bilinear functional $\varphi: A \times$ $A \rightarrow \mathbb{C}$ satisfying (1.1), there exists $\omega \in A^{*}$ such that (1.2) holds. This notion, introduced in our recent paper [4, can be viewed as the Lie analogue of the notion of a Banach algebra with property $\mathbb{B}$, introduced in [2] and subsequently studied in several papers (see [5] and references therein).

The main result of [4] states that the group algebra $L^{1}(G)$, where $G$ is an amenable locally compact group, is zero Lie product determined. In Section 2 we take a different approach and in particular show that this is actually true for every locally compact group $G$. The main result (Theorem 2.7) shows much more, namely, that every weakly amenable Banach algebra with property $\mathbb{B}$ and a bounded approximate identity satisfies a certain generalized version of the zero Lie product determined property. Yet another approach is taken in Section 3, connecting the problem of showing that a Banach algebra is zero Lie product determined with the problem of showing that a

2010 Mathematics Subject Classification. 43A20, 46H05, 46L05, 47B48.

Key words and phrases. Zero Lie product determined Banach algebra, property $\mathbb{B}$, weakly amenable Banach algebra, cyclically amenable Banach algebra, Jordan derivation.

The authors were supported by MINECO grant MTM2015-65020-P. The first, the third and the fourth named authors were supported by Junta de Andalucía grant FQM185. The second named author was supported by ARRS grant P1-0288. 
Jordan derivation is an (inner) derivation. It is interesting to note that each of the two approaches yields the fact that $C^{*}$-algebras are zero Lie product determined. This was also observed in [4], but derived as a simple corollary to a result by Goldstein [14]. Finally, in Section 4, we use the result of Section 3 to show that if $A$ is a cyclically amenable Banach algebra with a bounded approximate identity, then $M_{n}(A)$, the Banach algebra of $n \times n$ matrices (with $n \geq 2$ ) over $A$, is zero Lie product determined.

\section{WEAK AMENABILITY AND PROPERTY $\mathbb{B}$}

Recall that a Banach algebra $A$ is said to be weakly amenable if every continuous derivation from $A$ into $A^{*}$ is inner. For a thorough treatment of this property and an account of many interesting examples of weakly amenable Banach algebras we refer the reader to [11]. We should remark that each $C^{*}$-algebra and the group algebra $L^{1}(G)$ of each locally compact group $G$ are weakly amenable [11, Theorems 5.6.48 and 5.6.77]. If the Banach algebra $A$ is commutative, then $A^{*}$ is a commutative Banach $A$-bimodule and therefore a derivation from $A$ into $A^{*}$ is inner if and only if it is zero. It is worth pointing out that a basic obstruction to the weak amenability is the existence of non-zero, continuous point derivations [11, Theorem 2.8.63(ii)]. Recall that a linear functional $D$ on $A$ is a point derivation at a given multiplicative linear functional $f$ if

$$
D(a b)=D(a) f(b)+f(a) D(b) \quad(a, b \in A) .
$$

We say that a Banach algebra $A$ has property $\mathbb{B}$ if for every continuous bilinear functional $\varphi: A \times A \rightarrow \mathbb{C}$, the condition

$$
a, b \in A, a b=0 \Longrightarrow \varphi(a, b)=0
$$

implies the condition

$$
\varphi(a b, c)=\varphi(a, b c) \quad(a, b, c \in A) .
$$

In [2] it was shown that many important examples of Banach algebras, including $C^{*}$-algebras and group algebras $L^{1}(G)$ where $G$ is any locally compact group, have property $\mathbb{B}$.

For a Banach space $X$, we denote by $\mathcal{B}(X)$ the Banach algebra of bounded linear operators on $X$, and by $\mathcal{A}(X)$ the closed ideal of $\mathcal{B}(X)$ consisting of approximable operators.

Proposition 2.1. Let $X$ be a Banach space. Then:

(1) The Banach algebra $\mathcal{A}(X)$ has property $\mathbb{B}$;

(2) The Banach algebra $\mathcal{B}\left(X^{n}\right)$ has property $\mathbb{B}$ for each $n \in \mathbb{N}$ with $n \geq 2$.

Proof. From [2, Example 1.3.3 and Theorem 2.11] we see that $\mathcal{A}(X)$ has property $\mathbb{B}$. The Banach algebra $\mathcal{B}\left(X^{n}\right)$ is isomorphic to the Banach algebra $M_{n}(\mathcal{B}(X))$ of all $n \times n$ matrices with entries in $\mathcal{B}(X)$. By 5 , Proposition 4.2], $M_{n}(\mathcal{B}(X))$ is generated by idempotents and [2, Example 1.3.3 and Theorem 2.11] then show that it has property $\mathbb{B}$.

Remark 2.2. Given a Banach space $X$, and $1 \leq p \leq \infty$, we write $\ell^{p}(X)$ for the Banach space of sequences $\left(x_{n}\right)$ in $X$ so that $\left(\left\|x_{n}\right\|\right) \in \ell^{p}$ with norm given by $\left\|\left(x_{n}\right)\right\|=\left\|\left(\left\|x_{n}\right\|\right)\right\|_{p}$. Since $\ell^{p}(X)$ is isomorphic to $\ell^{p}(X) \oplus \ell^{p}(X)$, 
it follows that the Banach algebra $\mathcal{B}\left(\ell^{p}(X)\right)$ has property $\mathbb{B}$. In particular, the Banach algebra $\mathcal{B}\left(\ell^{p}\right)$ has property $\mathbb{B}$.

Proposition 2.3. Let $A$ be a Banach algebra.

(1) If $A$ is essential and has property $\mathbb{B}$, then there are no non-zero, continuous point derivations on $A$.

(2) If $A$ is unital and contains a closed one-codimensional ideal I such that $I^{2}$ is not dense in $I$, then there is a non-zero, continuous point derivation on $A$.

Proof. (1) Since $A$ is essential there are no non-zero, continuous point derivations on $A$ at 0 . Let $D \in A^{*}$ be a continuous point derivation at a character $f$ of $A$. We define a continuous bilinear functional $\varphi: A \times A \rightarrow \mathbb{C}$ by $\varphi(a, b)=f(a) D(b)$ for all $a, b \in A$. We claim that $\varphi$ satisfies (2.2). Suppose that $a, b \in A$ are such that $a b=$ 0 . Then $f(a) f(b)=0$. If $f(a)=0$, then clearly $\varphi(a, b)=0$, as claimed. If $f(a) \neq 0$, then $f(b)=0$ and (2.1) yields $\varphi(a, b)=$ $D(a b)-D(a) f(b)=0$, as claimed. Let $a \in A$ be such that $f(a)=1$. Since $A$ has property $\mathbb{B},(2.3)$ holds and we have

$$
\begin{aligned}
f(b) D(a) & =f(a b) D(a)=\varphi(a b, a)=\varphi(a, b a) \\
& =f(a) D(b a)=D(b a)=D(b)+f(b) D(a),
\end{aligned}
$$

which gives $D(b)=0$ for each $b \in A$.

(2) Let $f$ be a character of $A$ such that $\operatorname{ker}(f)=I$, and let $x \in I$ be such that $x \notin \overline{I^{2}}$. Then $M=\mathbb{C} \mathbf{1}+\overline{I^{2}}$ is a closed subspace of $A$ and $x \notin M$. Hence there exists $D \in A^{*}$ such that $D(M)=\{0\}$ and $D(x)=1$. By [11, Proposition 2.7.11(i)], $D$ is a non-zero, continuous point derivation at $f$.

Corollary 2.4. There exists a Banach space $X$ such that $\mathcal{B}(X)$ does not have property $\mathbb{B}$.

Proof. An example of a Banach space $X$ such that $\mathcal{B}(X)$ contains an ideal $I$ satisfying the conditions of the second assertion of Proposition 2.3 is given in [19. Thus there exists a non-zero, continuous point derivation on $\mathcal{B}(X)$. The first assertion of Proposition 2.3 then establishes the result.

The following result is stated in [3] as Theorem 2.2, but only for the case where $A$ is a $C^{*}$-algebra. From the proof, however, it is clear that it holds for every Banach algebra with property $\mathbb{B}$ that has a bounded approximate identity. See also [4, Lemma 4.1] which covers the general case, only the (easy) part concerning the functional $\tau$ is missing.

Lemma 2.5. 3 Let $A$ be a Banach algebra with property $\mathbb{B}$ and having a bounded approximate identity, and let $\varphi: A \times A \rightarrow \mathbb{C}$ be a continuous bilinear map satisfying the condition:

$$
a, b \in A, a b=b a=0 \Longrightarrow \varphi(a, b)=0 .
$$

Then there exists $\sigma \in A^{*}$ such that

$$
\varphi(a b, c)-\varphi(b, c a)+\varphi(b c, a)=\sigma(a b c) \quad(a, b, c \in A) .
$$


Moreover, there exists $\tau \in A^{*}$ such that

$$
\varphi(a, b)+\varphi(b, a)=\tau(a \circ b) \quad(a, b \in A) .
$$

In the next lemma we take the conclusion of Lemma 2.5 as an assumption. But first, recall that given any Banach algebra $A$, the dual space $A^{*}$ of $A$ becomes a Banach $A$-bimodule by setting

$$
(a \cdot f)(c)=f(c a), \quad(f \cdot a)(c)=f(a c) \quad\left(a, c \in A, f \in A^{*}\right) .
$$

Lemma 2.6. Let $A$ be any Banach algebra. If a skew-symmetric continuous bilinear map $\varphi: A \times A \rightarrow \mathbb{C}$ satisfies (2.4) for some $\sigma \in A^{*}$, then the map $\delta: A \rightarrow A^{*}$ defined by

$$
\delta(a)(c)=\varphi(a, c)+\frac{1}{2} \sigma(a \circ c)
$$

is a continuous derivation.

Proof. It is clear that $\delta$ is linear and continuous. We must show that it satisfies

$$
\delta(a b)=\delta(a) \cdot b+a \cdot \delta(b) \quad(a, b \in A) .
$$

We begin by noticing that (2.4) can be written as

$$
\sigma(a b c)=\varphi(a b, c)+\varphi(c a, b)+\varphi(b c, a) \quad(a, b, c \in A)
$$

for $\varphi$ is skew-symmetric. Note that this immediately yields

$$
\sigma(a b c)=\sigma(b c a) \quad(a, b, c \in A) .
$$

Now take arbitrary $a, b, c \in A$. We have

$$
\delta(a b)(c)=\varphi(a b, c)+\frac{1}{2} \sigma(a b \circ c) .
$$

From (2.6) it follows that

$$
\delta(a b)(c)=-\varphi(c a, b)-\varphi(b c, a)+\sigma(a b c)+\frac{1}{2} \sigma(a b c+c a b),
$$

and hence, in view of (2.7) and the skew-linearity of $\varphi$,

$$
\delta(a b)(c)=\varphi(b, c a)+\varphi(a, b c)+\sigma(b c a)+\frac{1}{2} \sigma(a b c+c a b) .
$$

Now compute the right-hand side of (2.5):

$$
\begin{aligned}
(\delta(a) \cdot b+a \cdot \delta(b))(c) & =\delta(a)(b c)+\delta(b)(c a) \\
& =\varphi(a, b c)+\frac{1}{2} \sigma(a \circ b c)+\varphi(b, c a)+\frac{1}{2} \sigma(b \circ c a) .
\end{aligned}
$$

Comparing this with (2.8), we see that (2.5) indeed holds.

As an easy consequence of Lemmas 2.5 and 2.6 we can now derive the following theorem.

Theorem 2.7. Let $A$ be a weakly amenable Banach algebra with property $\mathbb{B}$ and having a bounded approximate identity. If a continuous bilinear functional $\varphi: A \times A \rightarrow \mathbb{C}$ satisfies

$$
a, b \in A, a b=b a=0 \Longrightarrow \varphi(a, b)=0,
$$

then there exist $\tau_{1}, \tau_{2} \in A^{*}$ such that

$$
\varphi(a, b)=\tau_{1}(a b)+\tau_{2}(b a) \quad(a, b \in A) .
$$


Proof. Define $\varphi_{1}, \varphi_{2}: A \times A \rightarrow \mathbb{C}$ by

$$
\varphi_{1}(a, b)=\frac{1}{2}(\varphi(a, b)+\varphi(b, a)), \quad \varphi_{2}(a, b)=\frac{1}{2}(\varphi(a, b)-\varphi(b, a)) .
$$

Note that $\varphi=\varphi_{1}+\varphi_{2}$, and each $\varphi_{i}$ satisfies the condition of the theorem, i.e., $a b=b a=0$ implies $\varphi_{i}(a, b)=0$. Since $\varphi_{1}$ is symmetric it follows from Lemma 2.5 that

$$
2 \varphi_{1}(a, b)=\tau(a \circ b) \quad(a, b \in A)
$$

for some $\tau \in A^{*}$. It remains to consider $\varphi_{2}$ which is skew-symmetric. By Lemma 2.6 there exist $\sigma \in A^{*}$ and a continuous derivation $\delta: A \rightarrow A^{*}$ such that

$$
\delta(a)(c)=\varphi_{2}(a, c)+\frac{1}{2} \sigma(a \circ c) \quad(a, c \in A) .
$$

Since $A$ is weakly amenable, $\delta$ is inner. That is, $\delta(a)=a \cdot \omega-\omega \cdot a$ for some $\omega \in A^{*}$, and hence

$$
\omega(c a-a c)-\varphi_{2}(a, c)=\frac{1}{2} \sigma(a \circ c) \quad(a, c \in A) .
$$

Viewing this expression as a bilinear functional on $A \times A$, we see that the lefthand side is skew-symmetric and the right-hand side is symmetric. Therefore, both sides are zero. Thus

$$
\varphi_{2}(a, c)=\omega(c a-a c) \quad(a, c \in A) .
$$

From (2.9) and (2.10) we readily get the desired conclusion (with $\tau_{1}=\frac{\tau}{2}-\omega$ and $\left.\tau_{2}=\frac{\tau}{2}+\omega\right)$.

Corollary 2.8. Let $A$ be a weakly amenable Banach algebra $A$ with property $\mathbb{B}$ and having a bounded approximate identity. Then $A$ is a zero Lie product determined Banach algebra.

Proof. If $\varphi$ satisfies (1.1), then it is skew-symmetric and satisfies the assumption of Theorem 2.7. This clearly implies that $\varphi$ is of the desired form.

Every $C^{*}$-algebra satisfies all the assumptions of Corollary 2.8. We can thus state the following corollary which was derived in [4] from Goldstein's theorem [14].

Corollary 2.9. Every $C^{*}$-algebra is a zero Lie product determined Banach algebra.

Another type of a Banach algebra satisfying all the assumptions of Corollary 2.8 is $L^{1}(G)$ where $G$ is any locally compact group. Hence we have the following corollary. It is a substantial generalization of [4, Theorem 4.5] which covers only amenable groups.

Corollary 2.10. Let $G$ be a locally compact group. Then the group algebra $L^{1}(G)$ is a zero Lie product determined Banach algebra.

Corollary 2.11. Let $X$ be a Banach space, and $1 \leq p \leq \infty$. Then $\mathcal{B}\left(\ell^{p}(X)\right)$ is a zero Lie product determined Banach algebra. In particular, $\mathcal{B}\left(\ell^{p}\right)$ is a zero Lie product determined Banach algebra.

Proof. Proposition 2.1 and Remark 2.2 show that $\mathcal{B}\left(\ell^{p}(X)\right)$ has property $\mathbb{B}$. Further, [8, Proposition 3.2] shows that $\mathcal{B}\left(\ell^{p}(X)\right)$ is weakly amenable. Corollary 2.8 establishes our assertion. 


\section{CyClic Amenability And Jordan DERIVATIONS} if

Let $A$ be a Banach algebra. A derivation $D: A \rightarrow A^{*}$ is said to be cyclic

$$
D(a)(b)=-D(b)(a) \quad(a, b \in A) .
$$

Clearly inner derivations are cyclic. The algebra $A$ is said to be cyclically amenable if every continuous cyclic derivation $D: A \rightarrow A^{*}$ is inner. For an account of this notion we refer the reader to [15]. Of course, weak amenability implies cyclic amenability.

Let $A$ be a unital Banach algebra that is unitally polynomially generated by a single element $x$, and let $D: A \rightarrow A^{*}$ be a continuous cyclic derivation. We have

$$
D(\mathbf{1})=D\left(\mathbf{1}^{2}\right)=2(\mathbf{1} \cdot D(\mathbf{1}))=2 D(\mathbf{1}),
$$

which implies that $D(\mathbf{1})=0$. Further, $D(x)(\mathbf{1})=-D(\mathbf{1})(x)=0$ and, for $n \in \mathbb{N}$,

$$
D(x)\left(x^{n}\right)=-D\left(x^{n}\right)(x)=-\left(n x^{n-1} \cdot D(x)\right)(x)=-n D(x)\left(x^{n}\right),
$$

which yields $D(x)\left(x^{n}\right)=0$. This clearly implies that $D(x)(p(x))=0$ for each polynomial $p$ in one indeterminate, and hence $D(x)(a)=0$ for each $a \in A$. On the other hand, for each polynomial $p$ and each $a \in A$, we have

$$
D(p(x))(a)=\left(p^{\prime}(x) \cdot D(x)\right)(a)=D(x)\left(p^{\prime}(x) a\right)=0,
$$

and hence $D(b)(a)=0$ for each $b \in A$. Consequently, every cyclic derivation $D: A \rightarrow A^{*}$ is zero and therefore $A$ is cyclically amenable. Some relevant examples of unital Banach algebras that are unitally polynomially generated by a single element are the disc algebra $A(\mathbb{D}), C^{n}([a, b])$, and the algebra $A C([a, b])$ of absolutely continuous functions on the interval $[a, b]$ (see [11). It should be pointed out that none of these Banach algebras is weakly amenable. The Banach algebras $A(\mathbb{D})$ and $C^{n}([a, b])$ obviously have non-zero, continuous point derivations and so they are not weakly amenable (see [11, Theorem 2.8.63]). There are no non-zero continuous point derivations on $A C([a, b])$ but nevertheless this Banach algebra is not weakly amenable [11, Theorem 5.6.8]. The semigroup algebra $\ell^{1}\left(\mathbb{S}_{X}\right)$ of the free semigroup $\mathbb{S}_{X}$ generated by any set $X$ is cyclically amenable [15]. For the definition of the semigroup algebra we refer the reader to [11, Examples 2.1.13(iv),(v)]. However, the algebra $\ell^{1}\left(\mathbb{S}_{X}\right)$ is not essential. For, if $x \in X$, then there are no elements $s, t \in \mathbb{S}_{X}$ with $s t=x$, which implies that $(a \star b)(x)=0$ for all $a, b \in \ell^{1}\left(\mathbb{S}_{X}\right)$. From [11, Theorem 2.8.63(i)] it follows that the algebra $\ell^{1}\left(\mathbb{S}_{X}\right)$ is never weakly amenable, regardless of $X$.

Let $A$ be an algebra and $M$ an $A$-bimodule. For $a \in A$ and $m \in M$ we write $a \circ m=m \circ a=a \cdot m+m \cdot a$. Recall that a linear map $\delta: A \rightarrow M$ is called a Jordan derivation if it satisfies

$$
\delta(a \circ b)=\delta(a) \circ b+a \circ \delta(b) \quad(a, b \in A) .
$$

By the usual polarisation procedure, (3.1) is equivalent to

$$
\delta\left(a^{2}\right)=\delta(a) \cdot a+a \cdot \delta(a) \quad(a \in A) .
$$

Taking into account that, for all $a, b \in A$ and $m \in M$,

$$
2 a b a=(a \circ b) \circ a-a^{2} \circ b
$$


and

$$
2 a \cdot m \cdot a=(a \circ m) \circ a-a^{2} \circ m,
$$

it follows that every Jordan derivation $\delta$ satifies

$$
\delta(a b a)=\delta(a) \cdot(b a)+a \cdot \delta(b) \cdot a+(a b) \cdot \delta(a) .
$$

The question whether every Jordan derivation is actually a derivation has been studied by many authors in various contexts, starting in the 1950's. Roughly speaking, more often than not the answer is positive (see, e.g., [9]). The following result is therefore of some interest.

Theorem 3.1. Let $A$ be a Banach algebra. If every continuous cyclic Jordan derivation from $A$ into $A^{*}$ is an inner derivation, then $A$ is a zero Lie product determined Banach algebra.

Proof. Let $\varphi: A \times A \rightarrow \mathbb{C}$ be a continuous bilinear functional satisfying (1.1). In particular, $\varphi$ satisfies

$$
\varphi(a, a)=0 \quad \text { and } \quad \varphi\left(a^{2}, a\right)=0 \quad(a \in A) .
$$

Linearizing these identities we see that $\varphi$ is skew-symmetric and satisfies

$$
\varphi(a \circ b, c)+\varphi(c \circ a, b)+\varphi(b \circ c, a)=0 \quad(a, b, c \in A) .
$$

Define $\delta: A \rightarrow A^{*}$ by

$$
\delta(a)(c)=\varphi(a, c)
$$

for all $a, c \in A$. Applying (3.4), for all $a, b, c \in A$ we obtain

$$
\delta(a \circ b)(c)=\varphi(a \circ b, c)=\varphi(a, b \circ c)+\varphi(b, c \circ a) .
$$

From

$$
\begin{aligned}
(\delta(a) \circ b+a \circ \delta(b))(c) & =(\delta(a) \cdot b+b \cdot \delta(a)+a \cdot \delta(b)+\delta(b) \cdot a)(c) \\
& =\delta(a)(b c)+\delta(a)(c b)+\delta(b)(c a)+\delta(b)(a c) \\
& =\varphi(a, b \circ c)+\varphi(b, c \circ a)
\end{aligned}
$$

we thus see that $\delta$ is a Jordan derivation. Further, it is clear that $\delta$ is cyclic. By our hypothesis, it follows that $\delta$ is an inner derivation. Hence there exists $\omega \in A^{*}$ such that $\varphi(a, c)=\omega([c, a])$.

Corollary 3.2. Let $A$ be a cyclically amenable Banach algebra. If every continuous Jordan derivation from $A$ into $A^{*}$ is a derivation, then $A$ is a zero Lie product determined Banach algebra.

Remark 3.3. Johnson [18] proved that every continuous Jordan derivation from a $C^{*}$-algebra $A$ into any Banach $A$-bimodule $M$ is a derivation (see [6] for an alternative proof). Therefore Corollary 2.9 follows also from Corollary 3.2. In [18] it is also shown that every continuous Jordan derivation from the group algebra $L^{1}(G)$ into any Banach $L^{1}(G)$-bimodule $M$ is a derivation in the case where the group $G$ is amenable. This clearly implies that Corollary 2.10 follows from Corollary 3.2 in the case where the group is amenable. Nevertheless, to the best of our knowledge, it is not known whether every continuous Jordan derivation from $L^{1}(G)$ into $L^{1}(G)^{*}$ is actually a derivation for each locally compact group $G$. 
Proposition 3.4. Let $\mathbb{S}_{2}$ be the free semigroup with two generators. Then there exists a continuous Jordan derivation from $\ell^{1}\left(\mathbb{S}_{2}\right)$ into $\ell^{1}\left(\mathbb{S}_{2}\right)^{*}$ which is not a derivation.

Proof. Let $x_{1}$ and $x_{2}$ be the generators of $\mathbb{S}_{2}$, and let $u_{1}, u_{2} \in M_{4}(\mathbb{C})$ be the matrices given by

$$
u_{1}=\left(\begin{array}{cccc}
0 & 1 & 0 & 0 \\
0 & 0 & 0 & 0 \\
0 & 0 & 0 & -1 \\
0 & 0 & 0 & 0
\end{array}\right), u_{2}=\left(\begin{array}{cccc}
0 & 0 & 1 & 0 \\
0 & 0 & 0 & 1 \\
0 & 0 & 0 & 0 \\
0 & 0 & 0 & 0
\end{array}\right) .
$$

For each word $w \in \mathbb{S}_{2}$ let $\delta_{w} \in \ell^{1}\left(\mathbb{S}_{2}\right)$ stands for the characteristic function of the set $\{w\}$. Further, each element $w \in \mathbb{S}_{2}$ has the form $w=x_{j_{1}}^{k_{1}} \cdots x_{j_{n}}^{k_{n}}$ where $n, k_{1}, \ldots, k_{n} \in \mathbb{N}$ and $j_{1}, \ldots, j_{n} \in\{1,2\}$, and, since $\left\|u_{1}\right\|=\left\|u_{2}\right\|=1$, there exists a unique continuous algebra homomorphism $\Phi: \ell^{1}\left(\mathbb{S}_{2}\right) \rightarrow M_{4}(\mathbb{C})$ such that

$$
\Phi\left(\delta_{x_{j_{1}}^{k_{1} \ldots j_{j_{n}}^{k_{n}}}}\right)=u_{j_{1}}^{k_{1}} \cdots u_{j_{n}}^{k_{n}} \quad\left(n, k_{1}, \ldots, k_{n} \in \mathbb{N}, j_{1}, \ldots, j_{n} \in\{1,2\}\right)
$$

Observe that $\Phi\left(\ell^{1}\left(\mathbb{S}_{2}\right)\right)$ is the subalgebra of $M_{4}(\mathbb{C})$ generated by $u_{1}$ and $u_{2}$ and that

$$
u_{1}^{2}=u_{2}^{2}=u_{1} u_{2}+u_{2} u_{1}=0 .
$$

This implies that $\Phi(a \circ b)=0$ for all $a, b \in \ell^{1}\left(\mathbb{S}_{2}\right)$. We also define $\tau: M_{4}(\mathbb{C}) \rightarrow$ $\mathbb{C}$ by $\tau(u)=u_{12}+u_{14}\left(u \in M_{4}(\mathbb{C})\right)$, and finally $D: \ell^{1}\left(\mathbb{S}_{2}\right) \rightarrow \ell^{1}\left(\mathbb{S}_{2}\right)^{*}$ by

$$
D(a)(b)=\tau(\Phi(a)) \tau(\Phi(b)) \quad\left(a, b \in \ell^{1}\left(\mathbb{S}_{2}\right)\right) .
$$

We claim that $D$ is a Jordan derivation. If $a, b \in \ell^{1}\left(\mathbb{S}_{2}\right)$, then $\Phi\left(a^{2}\right)=0$, which yields $D\left(a^{2}\right)(b)=0$, and

$$
(D(a) \cdot a+a \cdot D(a))(b)=D(a)(a \circ b)=\tau(\Phi(a)) \tau(\Phi(a \circ b))=0 .
$$

We now show that $D$ is not a derivation. Indeed,

$$
D\left(\delta_{x_{1}} \star \delta_{x_{2}}\right)\left(\delta_{x_{1}}\right)=D\left(\delta_{x_{1} x_{2}}\right)\left(\delta_{x_{1}}\right)=1,
$$

while

$$
\left(D\left(\delta_{x_{1}}\right) \cdot \delta_{x_{2}}+\delta_{x_{1}} \cdot D\left(\delta_{x_{2}}\right)\right)\left(\delta_{x_{1}}\right)=D\left(\delta_{x_{1}}\right)\left(\delta_{x_{2} x_{1}}\right)+D\left(\delta_{x_{2}}\right)\left(\delta_{x_{1}^{2}}\right)=-1 .
$$

Question 3.5. Is the semigroup algebra $\ell^{1}\left(\mathbb{S}_{2}\right)$ a zero Lie product determined Banach algebra? Since $\ell^{1}\left(\mathbb{S}_{2}\right)$ is cyclically amenable it suffices to find out whether every continuous cyclic Jordan derivation from $\ell^{1}\left(\mathbb{S}_{2}\right)$ into $\ell^{1}\left(\mathbb{S}_{2}\right)^{*}$ is a derivation. Proposition 3.4 indicates the delicacy of this problem.

Remark 3.6. Let $X$ be a Banach space. On account of [18, Theorem 6.4], every continuous Jordan derivation from $\mathcal{A}(X)$ into any Banach $\mathcal{A}(X)$ bimodule is a derivation. On the other hand, we refer the reader to [7, 12, 16, 17] for a deep discussion of the weak amenability of $\mathcal{A}(X)$. It is worth pointing out that $\mathcal{A}\left(\ell^{p}(X)\right)$ is weakly amenable for each Banach space $X$ having the bounded approximation property and $1 \leq p<\infty$ (see [17, Corollary 4.3]).

The preceding remark together with Theorem 3.1 yield the following. 
Corollary 3.7. Let $X$ be a Banach space having the bounded approximation property, and $1 \leq p<\infty$. Then $\mathcal{A}\left(\ell^{p}(X)\right)$ is a zero Lie product determined Banach algebra. In particular, $\mathcal{A}\left(\ell^{p}\right)$ is a zero Lie product determined Banach algebra.

\section{Matrix Algebras}

Let $A$ be a Banach algebra, and let $n \in \mathbb{N}$. By $M_{n}(A)$ we denote the Banach algebra of $n \times n$ matrices with entries in $A$. Let $A^{\sharp}$ denote the algebra formed by adjoining an identity to $A$ as defined in [11, Definition 1.3.3]. For $i, j \in\{1, \ldots, n\}$ and $a \in A^{\sharp}$, let $a E_{i j} \in M_{n}\left(A^{\sharp}\right)$ be the matrix with $a$ in the $(i, j)$ th position and 0 elsewhere. We abbreviate $1 E_{i j}$ to $E_{i j}$. Note that for every $\mathbf{a}=\left(a_{i j}\right) \in M_{n}\left(A^{\sharp}\right)$ we have

$$
E_{i j} \mathbf{a} E_{k l}=a_{j k} E_{i l} \quad(1 \leq i, j, k, l \leq n) .
$$

The algebra $M_{n}(A)$ is a closed two-sided ideal of $M_{n}\left(A^{\sharp}\right)$ and therefore $M_{n}(A)$ is a Banach $M_{n}(\mathbb{C} 1)$-bimodule. From now on, we identify $M_{n}(\mathbb{C} 1)$ with $M_{n}(\mathbb{C})$ in the natural way.

Lemma 4.1. Let $A$ be a Banach algebra with a bounded approximate identity, and let $D: A \rightarrow A^{*}$ be a continuous Jordan derivation. Suppose that $B$ is a Banach algebra which contains $A$ as a closed two-sided ideal. Then there exists a continuous Jordan derivation $\Delta: B \rightarrow A^{*}$ which extends $D$.

Proof. Let $\left(u_{\lambda}\right)_{\lambda \in \Lambda}$ be an approximate identity for $A$ of bound $C$, and let $\mathcal{U}$ be an ultrafilter on $\Lambda$ refining the order filter. Let $a \in A$ and $x \in B$. For every $\lambda \in \Lambda$, we have

$$
\left|D\left(u_{\lambda} x u_{\lambda}\right)(a)\right| \leq\|D\|\left\|u_{\lambda} x u_{\lambda}\right\|\|a\| \leq C^{2}\|D\|\|x\|\|a\| .
$$

Therefore the net of complex numbers $\left(D\left(u_{\lambda} x u_{\lambda}\right)(a)\right)_{\lambda \in \Lambda}$ is bounded by $C^{2}\|D\|\|x\|\|a\|$ and so it has a unique limit along the ultrafilter $\mathcal{U}$. Hence we can define $\Delta: B \rightarrow A^{*}$ by

$$
\Delta(x)(a)=\lim _{\mathcal{U}} D\left(u_{\lambda} x u_{\lambda}\right)(a)
$$

for all $x \in B$ and $a \in A$. Indeed, it is routine that $\Delta(x) \in A^{*}$ with $\|\Delta(x)\| \leq$ $C^{2}\|D\|\|x\|$ and that $\Delta$ is a continuous linear map with $\|\Delta\| \leq C^{2}\|D\|$.

We claim that $\Delta$ extends $D$. If $b \in A$, then $\left(u_{\lambda} b u_{\lambda}\right)_{\lambda \in \Lambda} \rightarrow b$ in norm and the continuity of $D$ gives $\left(D\left(u_{\lambda} b u_{\lambda}\right)\right)_{\lambda \in \Lambda} \rightarrow D(b)$. We thus get

$$
\Delta(b)(a)=\lim _{\mathcal{U}} D\left(u_{\lambda} b u_{\lambda}\right)(a)=D(b)(a)
$$

for each $a \in A$.

Our next goal is to show that $\Delta$ is a Jordan derivation. By (3.3) we have

$$
\begin{aligned}
D\left(u_{\lambda} x^{2} u_{\lambda}\right)(b c b)= & \left(b \cdot D\left(u_{\lambda} x^{2} u_{\lambda}\right) \cdot b\right)(c) \\
= & D\left(b\left(u_{\lambda} x^{2} u_{\lambda}\right) b\right)(c) \\
& -\left(D(b) \cdot\left(\left(u_{\lambda} x^{2} u_{\lambda}\right) b\right)\right)(c)-\left(\left(b\left(u_{\lambda} x^{2} u_{\lambda}\right)\right) \cdot D(b)\right)(c) \\
= & D\left(b u_{\lambda} x^{2} u_{\lambda} b\right)(c)-D(b)\left(u_{\lambda} x^{2} u_{\lambda} b c\right)-D(b)\left(c b u_{\lambda} x^{2} u_{\lambda}\right) .
\end{aligned}
$$


Since $\left\|b u_{\lambda}-b\right\| \rightarrow 0$ and $\left\|u_{\lambda} b-b\right\| \rightarrow 0$, it follows that $\left\|b u_{\lambda} x^{2} u_{\lambda} b-b x^{2} b\right\| \rightarrow 0$, and the continuity of $D$ then gives

$$
\lim _{\mathcal{U}} D\left(b u_{\lambda} x^{2} u_{\lambda} b\right)(c)=D\left(b x^{2} b\right)(c) .
$$

On the other hand,

$$
\begin{aligned}
\left\|u_{\lambda} x^{2} u_{\lambda} b c-x^{2} b c\right\| & \leq\left\|u_{\lambda} x^{2} u_{\lambda} b c-u_{\lambda} x^{2} b c\right\|+\left\|u_{\lambda} x^{2} b c-x^{2} b c\right\| \\
& \leq\left\|u_{\lambda} x^{2}\right\|\left\|u_{\lambda}(b c)-b c\right\|+\left\|u_{\lambda}\left(x^{2} b c\right)-x^{2} b c\right\| \\
& \leq C\left\|x^{2}\right\|\left\|u_{\lambda}(b c)-b c\right\|+\left\|u_{\lambda}\left(x^{2} b c\right)-x^{2} b c\right\| \rightarrow 0,
\end{aligned}
$$

and the continuity of the functional $D(b)$ now gives

$$
\lim _{\mathcal{U}} D(b)\left(u_{\lambda} x^{2} u_{\lambda} b c\right)=D(b)\left(x^{2} b c\right) .
$$

By a similar argument we prove that

$$
\lim _{\mathcal{U}}\left\|c b u_{\lambda} x^{2} u_{\lambda}-c b x^{2}\right\|=0
$$

and hence that

$$
\lim _{\mathcal{U}}\left(D(b)\left(c b u_{\lambda} x^{2} u_{\lambda}\right)\right)_{\lambda \in \Lambda}=D(b)\left(c b x^{2}\right) .
$$

We thus get

$$
\Delta\left(x^{2}\right)(b c b)=D\left(b x^{2} b\right)(c)-D(b)\left(x^{2} b c\right)-D(b)\left(c b x^{2}\right) .
$$

In much the same way we get

$$
\begin{aligned}
D\left(\left(u_{\lambda} x u_{\lambda}\right)^{2}\right)(b c b)= & D\left(u_{\lambda} x u_{\lambda}^{2} x u_{\lambda}\right)(b c b) \\
= & \left(b \cdot D\left(u_{\lambda} x u_{\lambda}^{2} x u_{\lambda}\right) \cdot b\right)(c) \\
= & D\left(b\left(u_{\lambda} x u_{\lambda}^{2} x u_{\lambda}\right) b\right)(c)-\left(D(b) \cdot\left(\left(u_{\lambda} x u_{\lambda}^{2} x u_{\lambda}\right) b\right)\right)(c) \\
& -\left(\left(b\left(u_{\lambda} x u_{\lambda}^{2} x u_{\lambda}\right)\right) \cdot D(b)\right)(c) \\
= & D\left(b u_{\lambda} x u_{\lambda}^{2} x u_{\lambda} b\right)(c)-D(b)\left(u_{\lambda} x u_{\lambda}^{2} x u_{\lambda} b c\right) \\
& -D(b)\left(c b u_{\lambda} x u_{\lambda}^{2} x u_{\lambda}\right) \\
\rightarrow & D\left(b x^{2} b\right)(c)-D(b)\left(x^{2} b c\right)-D(b)\left(c b x^{2}\right) \\
= & \Delta\left(x^{2}\right)(b c b) .
\end{aligned}
$$

On the other hand, from (3.2) we deduce that

$$
\begin{aligned}
D\left(\left(u_{\lambda} x u_{\lambda}\right)^{2}\right)(b c b) & =\left(D\left(u_{\lambda} x u_{\lambda}\right) \cdot\left(u_{\lambda} x u_{\lambda}\right)+\left(u_{\lambda} x u_{\lambda}\right) \cdot D\left(u_{\lambda} x u_{\lambda}\right)\right)(b c b) \\
& =D\left(u_{\lambda} x u_{\lambda}\right)\left(u_{\lambda} x u_{\lambda} b c b+b c b u_{\lambda} x u_{\lambda}\right) .
\end{aligned}
$$

Write

and

$$
v_{\lambda}=u_{\lambda} x u_{\lambda} b c b+b c b u_{\lambda} x u_{\lambda}
$$

$$
v=x b c b+b c b x .
$$

Then $\left(v_{\lambda}\right)_{\lambda \in \Lambda} \rightarrow v$ in norm and therefore

$$
\begin{aligned}
\left|D\left(u_{\lambda} x u_{\lambda}\right)\left(v_{\lambda}\right)-D\left(u_{\lambda} x u_{\lambda}\right)(v)\right| & \leq\left\|D\left(u_{\lambda} x u_{\lambda}\right)\right\|\left\|v_{\lambda}-v\right\| \\
& \leq C^{2}\|D\|\|x\|\left\|v_{\lambda}-v\right\| \rightarrow 0 .
\end{aligned}
$$


Since $\lim _{\mathcal{U}} D\left(u_{\lambda} x u_{\lambda}\right)(v)=\Delta(x)(v)$, it may be concluded that

$$
\begin{aligned}
\lim _{\mathcal{U}} D\left(\left(u_{\lambda} x u_{\lambda}\right)^{2}\right)(b c b) & =\lim _{\mathcal{U}} D\left(u_{\lambda} x u_{\lambda}\right)\left(v_{\lambda}\right)=\Delta(x)(v) \\
& =\Delta(x)(x b c b+b c b x) \\
& =(\Delta(x) \cdot x+x \cdot \Delta(x))(b c b) .
\end{aligned}
$$

From what has been proved and (4.2) we deduce that

$$
\left(\Delta\left(x^{2}\right)-\Delta(x) \cdot x-x \cdot \Delta(x)\right)(b c b)=0
$$

for all $b, c \in A$. Let $a \in A$. Then [1, Théorème II.16] gives $b, c \in A$ such that $a=b c b$ and so

$$
\left(\Delta\left(x^{2}\right)-\Delta(x) \cdot x-x \cdot \Delta(x)\right)(a)=0 .
$$

This entails that $\Delta\left(x^{2}\right)=\Delta(x) \cdot x+x \cdot \Delta(x)$.

Corollary 4.2. Let $A$ be a Banach algebra with a bounded approximate identity, and let $D: M_{n}(A) \rightarrow M_{n}(A)^{*}$, where $n \geq 2$, be a continuous Jordan derivation. Then $D$ is a derivation and there exists a continuous derivation $\Delta: M_{n}\left(A^{\sharp}\right) \rightarrow M_{n}(A)^{*}$ which extends $D$.

Proof. Let $\left(u_{\lambda}\right)_{\lambda \in \Lambda}$ be a bounded approximate identity for $A$ and, for each $\lambda \in \Lambda$, take $\mathbf{u}_{\lambda}$ to be the matrix with $u_{\lambda}$ in the diagonal and 0 elsewhere. Then $\left(\mathbf{u}_{\lambda}\right)_{\lambda \in \Lambda}$ is a bounded approximate identity for $M_{n}(A)$. Further, $M_{n}(A)$ is a closed two-sided ideal of $M_{n}\left(A^{\sharp}\right)$. According to Lemma 4.1, there exists a continuous Jordan derivation $\Delta: M_{n}\left(A^{\sharp}\right) \rightarrow M_{n}(A)^{*}$ which extends $D$. By [18, Theorem 7.1], $\Delta$ is a derivation and this proves the result.

Lemma 4.3. Let $A$ be a cyclically amenable Banach algebra with a bounded approximate identity. Then $M_{n}(A)$ is cyclically amenable.

Proof. Let $D: M_{n}(A) \rightarrow M_{n}(A)^{*}$ be a continuous cyclic derivation.

From Corollary 4.2 it follows that there exists a continuous derivation $\Delta: M_{n}\left(A^{\sharp}\right) \rightarrow M_{n}(A)^{*}$ which extends $D$. We now consider the restriction of $\Delta$ to the subalgebra $M_{n}(\mathbb{C})$ of $M_{n}\left(A^{\sharp}\right)$. This gives a derivation from $M_{n}(\mathbb{C})$ to the Banach $M_{n}(\mathbb{C})$-bimodule $M_{n}(A)^{*}$. Since every derivation from $M_{n}(\mathbb{C})$ to any Banach $M_{n}(\mathbb{C})$-bimodule is inner (see [11, Example 1.9.23]), we conclude that there exists a functional $F_{1} \in M_{n}(A)^{*}$ such that

$$
\Delta(\alpha)=\alpha \cdot F_{1}-F_{1} \cdot \alpha \quad\left(\alpha \in M_{n}(\mathbb{C})\right),
$$

i.e.,

$$
\Delta(\alpha)(\mathbf{a})=F_{1}(\mathbf{a} \alpha-\alpha \mathbf{a}) \quad\left(\alpha \in M_{n}(\mathbb{C}), \mathbf{a} \in M_{n}(A)\right) .
$$

The map $D_{0}: M_{n}(A) \rightarrow M_{n}(A)^{*}$ defined by

$$
D_{0}(\mathbf{a})=D(\mathbf{a})-\left(\mathbf{a} \cdot F_{1}-F_{1} \cdot \mathbf{a}\right) \quad\left(\mathbf{a} \in M_{n}(A)\right)
$$

is a continuous cyclic derivation. We claim that

$$
D_{0}(\alpha \mathbf{a} \beta)=\alpha D_{0}(\mathbf{a}) \beta \quad\left(\alpha, \beta \in M_{n}(\mathbb{C}), \mathbf{a} \in M_{n}(A)\right) .
$$


Indeed, if $\alpha, \beta \in M_{n}(\mathbb{C})$ and $\mathbf{a} \in M_{n}(A)$, then

$$
\begin{aligned}
D_{0}(\alpha \mathbf{a} \beta)= & D(\alpha \mathbf{a} \beta)-(\alpha \mathbf{a} \beta) \cdot F_{1}+F_{1} \cdot(\alpha \mathbf{a} \beta) \\
= & \Delta(\alpha \mathbf{a} \beta)-(\alpha \mathbf{a} \beta) \cdot F_{1}+F_{1} \cdot(\alpha \mathbf{a} \beta) \\
= & \Delta(\alpha) \cdot(\mathbf{a} \beta)+\alpha \cdot \Delta(\mathbf{a}) \cdot \beta+(\alpha \mathbf{a}) \cdot \Delta(\beta) \\
& -(\alpha \mathbf{a} \beta) \cdot F_{1}+F_{1} \cdot(\alpha \mathbf{a} \beta) \\
= & \left(\alpha \cdot F_{1}-F_{1} \cdot \alpha\right)(\mathbf{a} \beta)+\alpha \cdot D(\mathbf{a}) \cdot \beta+(\alpha \mathbf{a}) \cdot\left(\beta \cdot F_{1}-F_{1} \cdot \beta\right) \\
& -(\alpha \mathbf{a} \beta) \cdot F_{1}+F_{1} \cdot(\alpha \mathbf{a} \beta) \\
= & \alpha \cdot F_{1} \cdot(\mathbf{a} \beta)+\alpha \cdot D(\mathbf{a}) \cdot \beta-(\alpha \mathbf{a}) \cdot F_{1} \cdot \beta \\
= & \alpha \cdot\left(D(\mathbf{a})-\mathbf{a} \cdot F_{1}+F_{1} \cdot \mathbf{a}\right) \cdot \beta \\
= & \alpha \cdot D_{0}(\mathbf{a}) \cdot \beta .
\end{aligned}
$$

We now define a continuous linear map $d: A \rightarrow A^{*}$ by

$$
d(a)(b)=D_{0}\left(a E_{11}\right)\left(b E_{11}\right) \quad(a, b \in A) .
$$

It is clear that $d$ cyclic. The task is now to show that $d$ is a derivation. Let $a, b, c \in A$. Then

$$
\begin{aligned}
d(a b)(c) & =D_{0}\left((a b) E_{11}\right)\left(c E_{11}\right)=D_{0}\left(\left(a E_{11}\right)\left(b E_{11}\right)\right)\left(c E_{11}\right) \\
& =\left(D_{0}\left(a E_{11}\right) \cdot\left(b E_{11}\right)+\left(a E_{11}\right) \cdot D\left(b E_{11}\right)\right)\left(c E_{11}\right) \\
& =D_{0}\left(a E_{11}\right)\left(\left(b E_{11}\right)\left(c E_{11}\right)\right)+D\left(b E_{11}\right)\left(\left(c E_{11}\right)\left(a E_{11}\right)\right) \\
& =D_{0}\left(a E_{11}\right)\left((b c) E_{11}\right)+D\left(b E_{11}\right)\left((c a) E_{11}\right) \\
& =d(a)(b c)+d(b)(c a) \\
& =(d(a) \cdot b+a \cdot d(b))(c) .
\end{aligned}
$$

Since $d$ is a continuous cyclic derivation and $A$ is cyclically amenable, it follows that there exists $f \in A^{*}$ such that

$$
d(a)=a \cdot f-f \cdot a \quad(a \in A),
$$

i.e.,

$$
d(a)(b)=f(b a-a b) \quad(a, b \in A) .
$$

We now define $F_{2} \in M_{n}(A)^{*}$ by

$$
F_{2}(\mathbf{a})=\sum_{k=1}^{n} f\left(a_{k k}\right) \quad\left(\mathbf{a}=\left(a_{i j}\right) \in M_{n}(A)\right)
$$

We claim that

$$
D_{0}(\mathbf{a})=\mathbf{a} \cdot F_{2}-F_{2} \cdot \mathbf{a} \quad\left(\mathbf{a} \in M_{n}(A)\right) .
$$


Let $\mathbf{a}=\left(a_{i j}\right), \mathbf{b}=\left(b_{i j}\right) \in M_{n}(A)$. Taking into account (4.1) and (4.4), we have

$$
\begin{aligned}
D_{0}(\mathbf{a})(\mathbf{b}) & =D_{0}\left(\sum_{i, j=1}^{n} a_{i j} E_{i j}\right)(\mathbf{b})=\sum_{i, j=1}^{n} D_{0}\left(a_{i j} E_{i j}\right)(\mathbf{b}) \\
& =\sum_{i, j=1}^{n} D_{0}\left(E_{i 1}\left(a_{i j} E_{11}\right) E_{1 j}\right)(\mathbf{b})=\sum_{i, j=1}^{n}\left(E_{i 1} \cdot D_{0}\left(a_{i j} E_{11}\right) \cdot E_{1 j}\right)(\mathbf{b}) \\
& =\sum_{i, j=1}^{n} D_{0}\left(a_{i j} E_{11}\right)\left(E_{1 j} \mathbf{b} E_{i 1}\right)=\sum_{i, j=1}^{n} D_{0}\left(a_{i j} E_{11}\right)\left(b_{j i} E_{11}\right) \\
& =\sum_{i, j=1}^{n} d\left(a_{i j}\right)\left(b_{j i}\right)=\sum_{i, j=1}^{n} f\left(b_{j i} a_{i j}-a_{i j} b_{j i}\right) \\
& =F_{2}(\mathbf{b a}-\mathbf{a b}) .
\end{aligned}
$$

According to (4.3) and (4.5), we have

$$
D(\mathbf{a})=\mathbf{a} \cdot F-F \cdot \mathbf{a} \quad\left(\mathbf{a} \in M_{n}(A)\right),
$$

where $F \in M_{n}(A)^{*}$ is defined by $F=F_{1}+F_{2}$, which proves that $D$ is inner as required.

Theorem 4.4. Let $A$ be a cyclically amenable Banach algebra with a bounded approximate identity, and $n \geq 2$. Then $M_{n}(A)$ is a zero Lie product determined Banach algebra.

Proof. By Corollary 4.2 and Lemma 4.3, $M_{n}(A)$ is cyclically amenable and every continuous Jordan derivation from $M_{n}(A)$ into $M_{n}(A)^{*}$ is a derivation. Corollary 3.2 completes the proof.

At this point it seems appropriate to mention a purely algebraic result from [10, stating that if a unital algebra $A$ is zero Lie product determined, then so is $M_{n}(A)$. (The definition of zero Lie product algebra is the same as that of zero Lie product Banach algebra, only the continuity is, of course, neglected.)

Example 4.5. Theorem 4.4 applies in any of the following cases.

(1) $A$ is a unital Banach algebra unitally polynomially generated by a single element.

(2) $A=L^{1}(G)$ where $G$ is any locally compact group.

(3) $A$ is a $C^{*}$-algebra. However, in this case $M_{n}(A)$ is itself a $C^{*}$-algebra and therefore Corollary 2.9 is applicable.

Remark 4.6. Let $A$ be a unital Banach algebra, and $n \geq 2$. By [5, Proposition 4.2], the Banach algebra $M_{n}(A)$ is generated by idempotents and [2, Example 1.3.3 and Theorem 2.11] then show that it has property $\mathbb{B}$. According to [13, Theorem 2.7(iii)], the Banach algebra $M_{n}(A)$ is weakly amenable if and only if $A$ is weakly amenable. Consequently, if $A$ is cyclically amenable but not weakly amenable (such as $A(\mathbb{D}), C^{n}([a, b]), A C([a, b])$ ), then the algebra $M_{n}(A)$ is not weakly amenable and cannot be handled by using Corollary 2.8 but Theorem 4.4 works. 


\section{REFERENCES}

[1] M. Akkar, M. Laayouni. Théorèmes de factorisation dans les algèbres completes de Jordan. Collect. Math. 46 (1995), 239-254.

[2] J. Alaminos, M. Brešar, J. Extremera, A. R. Villena, Maps preserving zero products, Studia Math. 193 (2009), 131-159.

[3] J. Alaminos, M. Brešar, J. Extremera, A. R. Villena, Characterizing Jordan maps on $C^{*}$-algebras through zero products, Proc. Edinburgh Math. Soc. 53 (2010), 543-555.

[4] J. Alaminos, M. Brešar, J. Extremera, A. R. Villena, Zero Lie product determined Banach algebras, Studia Math. 239 (2017), 189-199.

[5] J. Alaminos, M. Brešar, J. Extremera, Š, Špenko, A. R. Villena, Commutators and square-zero elements in Banach algebras, Quart. J. Math. 67 (2016), 1-13.

[6] J. Alaminos, M. Brešar, A. R. Villena, The strong degree of von Neumann algebras and the structure of Lie and Jordan derivations, Math. Proc. Cambridge Philos. Soc. 137 (2004), 441-463.

[7] A. Blanco, On the weak amenability of $\mathcal{A}(X)$ and its relation with the approximation property, J. Funct. Anal. 203 (2003), 1-26.

[8] A. Blanco, On the weak amenability of $\mathcal{B}(X)$, Studia Math. 196 (2010), 65-89.

[9] M. Brešar, Jordan derivations revisited, Math. Proc. Cambridge Philos. Soc. 139 (2005), 411-425.

[10] M. Brešar, M. Grašič, J. Sanchez, Zero product determined matrix algebras, Linear Algebra Appl. 430 (2009), 1486-1498.

[11] H.G. Dales, Banach algebras and automatic continuity, London Mathematical Society Monographs, New Series, 24, Oxford Science Publications, The Clarendon Press, Oxford University Press, New York, 2000.

[12] H. G. Dales, F. Ghahramani, and N. Grønbæk, Derivations into iterated duals of Banach algebras, Studia Math. 128 (1998), 19-54.

[13] H. G. Dales, A. T.-M. Lau, D. Strauss, Banach algebras on semigroups and on their compactifications. Mem. Amer. Math. Soc. 205 (2010), no. 966, vi+165 pp.

[14] S. Goldstein, Stationarity of operator algebras, J. Funct. Anal. 118 (1993), 275-308.

[15] N. Grønbæk, Weak and cyclic amenability for noncommutative Banach algebras, Proc. Edinburgh Math. Soc. 35 (1992), 315-328.

[16] N. Grønbæk, Factorization and weak amenability of algebras of approximable operators, Math. Proc. R. Ir. Acad. 106A (2006), 31-52.

[17] N. Grønbæk, Bounded Hochschild cohomology of Banach algebras with a matrix-like structure, Trans. Amer. Math. Soc. 358 (2006), 2651-2662.

[18] B. E. Johnson, Symmetric amenability and the nonexistence of Lie and Jordan derivations. Math. Proc. Cambridge Philos. Soc. 120 (1996), 455-473.

[19] C. J. Read, Discontinuous derivations on the algebra of bounded operators on a Banach space, J. London Math. Soc. 40 (1989), 305-326.

Departamento de Análisis Matemático, Facultad de Ciencias, Universidad De Granada, 18071 Granada, Spain

E-mail address: alaminos@ugr.es

Faculty of Mathematics and Physics, University of Ljubljana, Jadranska 19, 1000 Ljubljana, and, Faculty of Natural Sciences and Mathematics, UniVersity of Maribor, Koroška 160, 2000 Maribor, Slovenia

E-mail address: matej.bresar@fmf .uni-lj.si

Departamento de Análisis Matemático, Facultad de Ciencias, Universidad de Granada, 18071 Granada, Spain

E-mail address: jlizana@ugr.es

Departamento de Análisis Matemático, Facultad de Ciencias, Universidad De Granada, 18071 Granada, Spain

E-mail address: avillena@ugr.es 